\title{
Fano stability diagram of a symmetric triple quantum dot
}

\author{
Michael Niklas, Andreas Trottmann, Andrea Donarini, and Milena Grifoni* \\ Institute for Theoretical Physics, University of Regensburg, 93040 Regensburg, Germany \\ (Received 6 October 2016; revised manuscript received 10 February 2017; published 17 March 2017)
}

\begin{abstract}
The Fano factor stability diagram of a $C_{3 v}$ symmetric triangular quantum dot is analyzed for increasing electron fillings $N$. At low filling, conventional Poissonian and sub-Poissonian behavior is found. At larger filling, $N \geqslant 2$, a breaking of the electron-hole symmetry is manifested in super-Poissonian noise with a peculiar bias voltage dependence of the Fano factor at Coulomb and interference blockade. An analysis of the Fano map unravels a nontrivial electron-bunching mechanism arising from the presence of degenerate many-body states combined with orbital interference and Coulomb interactions. An expression for the associated dark states is provided for generic $N$.
\end{abstract}

DOI: 10.1103/PhysRevB.95.115133

\section{INTRODUCTION}

Current fluctuations in out-of-equilibrium nanoscale systems can yield information about relevant transport mechanisms not accessible from knowledge of only the average current [1]. In fermionic tunneling structures the interplay between the Pauli principle and repulsive Coulomb interactions usually yields Poissonian and sub-Poissonian noises, corresponding to Fano factors $F=1$ and $F<1$, respectively. For example, in single-level quantum dot systems one finds Poissonian shot noise at the Coulomb blockade [see Fig. 1(a)] and sub-Poissonian noise with $1 / 2<F<1$ in the sequential transport regime [2-7]. The latter is an indication that each tunneling barrier can be regarded as an independent source of Poissonian noise [8]. The enhancement of the shot noise, i.e., $F>1$, requires a multilevel structure of the quantum dot [8-10] or complex multiple-quantum-dot devices [11-15]. Independent of the details of the nanosystems, superPoissonian noise implies the presence of slow and fast channels and mechanisms which occasionally allow for charge transfer on a time scale much shorter than the average residence time in the slow-channel state [see, e.g., Figs. 1(b) and 1(c)]. Thus super-Poissonian noise is a signature of fermionic bunching and in turn of subtle quantum correlations, which is the topic of this work.

In this paper we investigate the Fano stability diagram of a $C_{3 v}$ symmetric triangular triple quantum dot (TQD), schematically sketched in Fig. 1(d), as a function of its occupation. TQDs are the smallest systems where the interplay of statistics, Coulomb interactions, and geometry allows one to study peculiar many-body effects such as superexchange-induced triplet-singlet transition [16], manybody interference $[13,17,18]$, cellular automata phenomena [19], charge frustration [20-22], and channel blockade [23]. TQDs have recently been realized in lateral semiconducting heterostructures $[19,20,23,24]$, which are tunable down to the few-electron regime by means of plunger and depletion gates [24] and by means of atomic scanning tunneling microscopy manipulation [25]. In the latter experiment, orbital degeneracy in a $C_{3 v}$ symmetric triangular dot could be demonstrated.

\footnotetext{
*Corresponding author: milena.grifoni@ur.de
}

So far, investigations of transport noise have been restricted to setups in which the $C_{3 v}$ symmetry of an isolated TQD is broken in various ways, e.g., by assuming unequal interdot hoppings and/or on-site energies [12,13,26-28]. These asymmetries remove orbital degeneracies and hence the possibility to observe current suppression due to the destructive orbital interference [29]. We show that the Fano map of a $C_{3 v}$ symmetric, weakly coupled TQD is characterized by regions of super-Poissonian noise outside the Coulomb blockade region [see Fig. 1(c)], with specific fractional values of the Fano factor being a signature of such many-body interference. By exploiting an analytical solution for the TQD spectrum, the explicit form of the dark states is provided, including the most complex situation of half filling.

\section{MODEL AND SPECTRUM}

We examine a single-electron transistor model described by the total Hamiltonian $H=H_{\mathrm{TQD}}+H_{\text {tun }}+H_{\text {res }}$. The TQD spectrum derives from a three-site Hamiltonian with hopping $b<0$, on-site Coulomb repulsion $U$, and intersite repulsion $V$,

$$
\begin{aligned}
H_{\mathrm{TQD}}= & \xi \sum_{i \sigma} n_{i \sigma}+b \sum_{i \neq j, \sigma} d_{j \sigma}^{\dagger} d_{i \sigma} \\
& +U \sum_{i}\left(n_{i \uparrow}-\frac{1}{2}\right)\left(n_{i \downarrow}-\frac{1}{2}\right) \\
& +V \sum_{i<j}\left(n_{i}-1\right)\left(n_{j}-1\right),
\end{aligned}
$$

where $\xi=\varepsilon-e \eta V_{\mathrm{g}}$ includes the on-site energy $\varepsilon$ and the effects of an applied gate voltage $V_{\mathrm{g}}$ with level arm $\eta$. Here, operators $d_{i \sigma}^{\dagger}$ and $d_{i \sigma}$ create and annihilate an electron with spin projection $\sigma$ in dot $i=0,1,2$, and $n_{i}=\sum_{\sigma} n_{i \sigma}$, $n_{i \sigma}=d_{i \sigma}^{\dagger} d_{i \sigma}$ [see Fig. 1(d)]. The two leads are considered reservoirs at chemical potentials $\mu_{L / R}=\mu_{0} \pm e V_{\mathrm{b}} / 2$ for the left $(L)$ and right $(R)$ leads, with $V_{\mathrm{b}}$ being the applied bias voltage, where we measure the energy from the equilibrium chemical potential $\mu_{0}=0$. The corresponding Hamiltonian is $H_{\text {res }}=\sum_{\alpha k \sigma} \xi_{\alpha k} c_{\alpha k \sigma}^{\dagger} c_{\alpha k \sigma}$, with $\alpha=L, R$. Finally, tunneling between the TQD and leads is described by $H_{\text {tun }}=\sum_{\alpha k \sigma} \sum_{i}\left(t_{\alpha i}^{*} c_{\alpha k \sigma}^{\dagger} d_{i \sigma}+t_{\alpha i} d_{i \sigma}^{\dagger} c_{\alpha k \sigma}\right)$. The single-orbital 

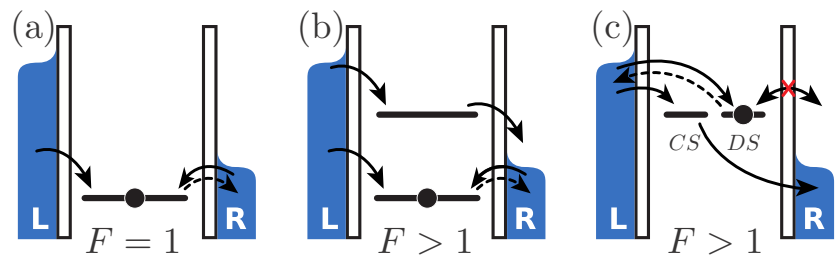

(d)

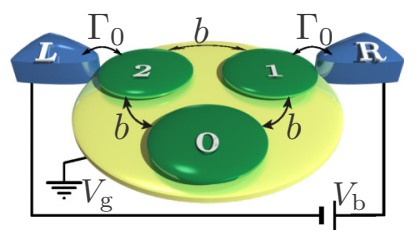

FIG. 1. (a) Single-level quantum dot in the Coulomb-blockade regime with Fano factor $F=1$. (b) If an excited state is present in the bias window of a Coulomb-blockaded quantum dot, electron bunching through the excited state yields super-Poissonian noise $(F>1)$. (c) Interference of energy degenerate orbitals gives rise to coupled (CS) and dark (DS) states and in turn to super-Poissonian noise. Solid arrows show fast processes; dashed ones show the dominant slow processes. (d) A gated triangular triple quantum dot (TQD) features all three dynamical situations sketched in (a)-(c).

approximation yields a realistic description of lateral TQD devices $[19,20,23,24]$ as long as $e V_{\mathrm{b}}$ and $e V_{\mathrm{g}}$ are of the order of the hopping parameter $b$ [30]. In the following, we consider equal coupling to the left and right leads and set $t_{L 2}=t_{R 1}=t$, and otherwise, $t_{\alpha i}=0$. We identify for later convenience $d_{1 \sigma}=d_{R \sigma}, d_{2 \sigma}=d_{L \sigma}$.

The single-particle part of the TQD Hamiltonian equation (1) is diagonalized in the basis of the angular momentum states $\left\{|l\rangle=1 / \sqrt{3} \sum_{j=0}^{2} e^{-i j l 2 \pi / 3}|j\rangle,\{l=0, \pm 1\}\right.$. Accounting for the spin degree of freedom $\sigma$, in the following we use this single-particle basis to construct many-body states in the occupation number representation, where a generic vector $\left|n_{0 \uparrow}, n_{1 \uparrow}, n_{-1 \uparrow} ; n_{0 \downarrow}, n_{1 \downarrow}, n_{-1 \downarrow}\right\rangle$ is fully characterized by the occupation numbers $n_{l \sigma}$. Finally, we use this many-body basis to diagonalize the TQD Hamiltonian and find its eigenvalues and eigenfunctions. Several symmetries have been exploited in the analytical diagonalization: $H_{\mathrm{TQD}}$ commutes, in fact, with the total particle number operator $N=\sum_{l \sigma} n_{l \sigma}$, the total spin operator $S^{2}=\sum_{i l \sigma \sigma^{\prime}}\left(d_{l \sigma}^{\dagger} s_{\sigma \sigma^{\prime}}^{i} d_{l \sigma^{\prime}}\right)^{2}$ (here $s^{i}=\frac{\hbar}{2} \sigma^{i}$ and $\sigma^{i}$ is the $i$ th Pauli matrix), the spin projection $S_{z}=\frac{\hbar}{2} \sum_{l \sigma} \sigma n_{l \sigma}$, and the angular momentum operator [17] $L_{z}=\left.\hbar \sum_{l \sigma} l_{l \sigma}\right|_{\bmod 3}$. By ordering the many-body states according to the quantum numbers $N, S, S_{z}$, and $L_{z}$ associated with these operators, we can reduce the Hamiltonian into a block-diagonal form with blocks of maximal size $3 \times 3$ and then complete the diagonalization. The set of eigenvalues listed above together with the energy $E$ fully characterize the eigenvectors of the interacting TQD Hamiltonian, which is crucial knowledge for the forthcoming analysis. In the following we use the notation $\left|N, E ; S, S_{z}, L_{z}\right\rangle$ or $\left|N, \alpha_{i}, L_{z}\right\rangle$, with $\alpha_{i}=\left\{E_{N_{i}} ; S, S_{z}\right\}$, for a generic eigenvector. In particular, $S^{2}\left|N, E ; S, S_{z}, L_{z}\right\rangle=$ $\hbar^{2} S(S+1)\left|N, E ; S, S_{z}, L_{z}\right\rangle$ and, as usual, $-S \leqslant S_{z} \leqslant S$. The $C_{3 v}$ group of the TQD also comprises three reflection planes perpendicular to the system. In particular, we introduce the reflection operator $\sigma_{v 0}$ such that $\sigma_{v 0} d_{1 \sigma}^{\dagger} \sigma_{v 0}=d_{2 \sigma}^{\dagger}$ and $\sigma_{v 0} d_{0 \sigma}^{\dagger} \sigma_{v 0}=d_{0 \sigma}^{\dagger}$. The overall phase of the eigenstates is taken in such a way that $\sigma_{v 0}\left|N, \alpha_{i}, 1\right\rangle=\left|N, \alpha_{i},-1\right\rangle$.

Such eigenvectors and the associated eigenvalues in the occupation number basis are reported in Appendix A. For convenience we set $\hbar=1$ in the quantum numbers.

\section{CURRENT AND NOISE IN A REDUCED DENSITY MATRIX APPROACH}

To compute the current and shot noise we use a masterequation approach for the generalized reduced density matrix $\rho_{\chi}=\operatorname{Tr}_{\text {res }}\left\{e^{i \chi N_{\mathrm{R}}} \rho\right\}$, where $\chi$ and $N_{\mathrm{R}}$ are the counting field and number operator for the right lead and $\rho$ is the total density operator [6,31]. A truncation to second order in $H_{\text {tun }}$ yields the generalized master equation $\dot{\rho}_{\chi}=$ $\left[\mathcal{L}+\left(e^{i \chi}-1\right) \mathcal{J}^{+}+\left(e^{-i \chi}-1\right) \mathcal{J}^{-}\right] \rho_{\chi}[32]$, where $\mathcal{L}$ is the Liouville superoperator, and we define the current superoperators for increasing, $\mathcal{J}^{+}$, and decreasing, $\mathcal{J}^{-}$, the number of electrons in the right lead. This results in the equations for the stationary reduced density matrix, $\rho^{\infty}=\lim _{t \rightarrow \infty} \rho_{\chi=0}$, and the moments $\mathcal{F}_{k}^{\infty}=\lim _{t \rightarrow \infty} d^{k} /\left.d(i \chi)^{k} \rho_{\chi}\right|_{\chi=0}$. Introducing the traceless part of the first moment, $\mathcal{F}_{1 \perp}^{\infty}=(1-$ $\left.\rho^{\infty} \operatorname{Tr}_{\mathrm{TQD}}\right) \mathcal{F}_{1}^{\infty}$, one finds in particular

$$
\begin{aligned}
\mathcal{L} \rho^{\infty} & =-\frac{i}{\hbar}\left[H_{\mathrm{TQD}}+H_{\mathrm{LS}}, \rho^{\infty}\right]+\mathcal{L}_{t} \rho^{\infty}=0, \\
\mathcal{L} \mathcal{F}_{1 \perp}^{\infty} & =\left(-I / e-\mathcal{J}^{+}+\mathcal{J}^{-}\right) \rho^{\infty},
\end{aligned}
$$

where $\mathcal{L}_{t}$ is the tunneling part of the Liouvillian. The Lamb shift Hamiltonian [18,33] $H_{\mathrm{LS}}$ generates a precession dynamics within orbitally degenerate subspaces. Its explicit form is given in Sec. VI. The operatorial form of Eq. (2) fully accounts for interference effects captured in the off-diagonal elements of $\rho^{\infty}$. The current $I$ (first cumulant) and shot noise $S$ (second cumulant) in turn follow as [32]

$$
\begin{aligned}
& I=-e \operatorname{Tr}_{\mathrm{TQD}}\left(\mathcal{J}^{+}-\mathcal{J}^{-}\right) \rho^{\infty}, \\
& S=e^{2} \operatorname{Tr}_{\mathrm{TQD}}\left[2\left(\mathcal{J}^{+}-\mathcal{J}^{-}\right) \mathcal{F}_{1 \perp}^{\infty}+\left(\mathcal{J}^{+}+\mathcal{J}^{-}\right) \rho^{\infty}\right] .
\end{aligned}
$$

As a dimensionless measure of the relative noise strength we employ the Fano factor $F=S / e|I|$.

\section{CURRENT AND FANO MAPS}

The stationary current is shown as a function of bias and gate voltage in Fig. 2(a). For comparison, the same parameters as in the work by Donarini et al. [29] are used. Notice that the closed geometry of the TQD breaks the particle-hole symmetry otherwise present in linear triple dots [16]. The stability diagram displays Coulomb diamonds inside of which current is exponentially suppressed (up to second order in $H_{\text {tun }}$ ) due to Coulomb blockade and also regions outside the Coulomb diamonds with suppression due to orbital interference [18]. Coulomb diamonds are indicated with dotted lines when no longer visible due to the additional interference blockade. A measurement of the current alone, however, does not enable one to tackle the different blockade mechanisms. In contrast, the Fano map, shown in Fig. 2(b), displays a much richer structure than the current. In Fig. 2(c) we show the Fano factor $F_{n v}$, which is obtained by neglecting the Lamb shift term $H_{\mathrm{LS}}$ 


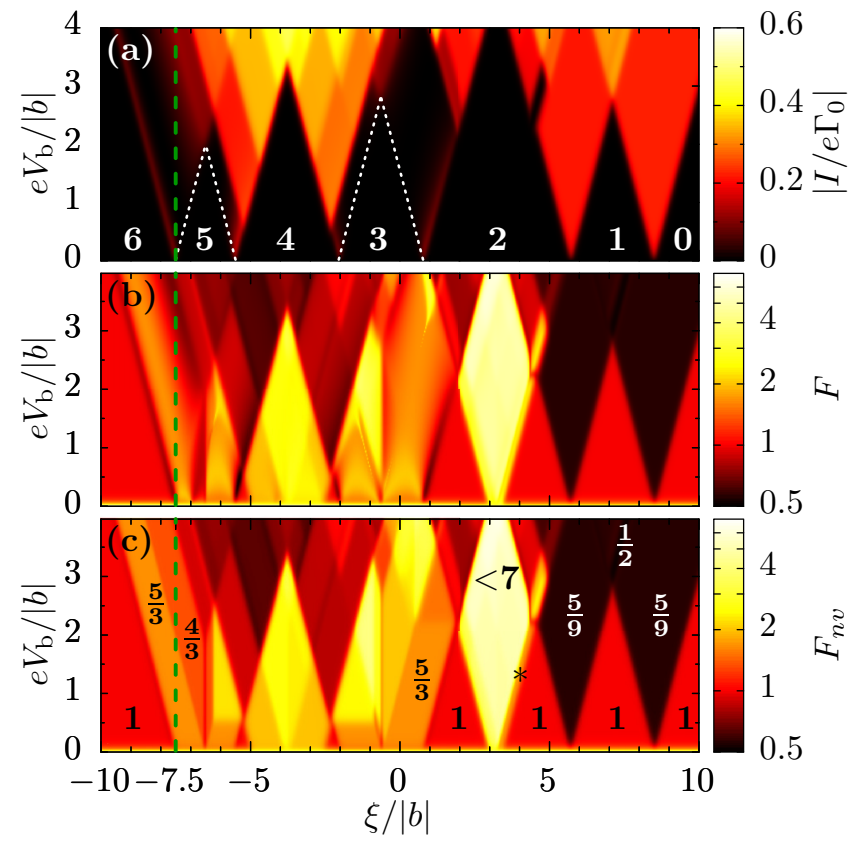

FIG. 2. Current and Fano factors vs applied gate and bias voltages. (a) Average current where the number of electrons in the blockade regions is displayed. The white dotted lines delimit regions where transport is inhibited due to Coulomb blockade. (b) Fano factor and (c) Fano factor without Lamb shifts due to virtual transitions. Some values discussed in the text are indicated. Parameters used for the simulations are $U=5|b|, V=2|b|, k_{\mathrm{B}} T=0.002|b|, k_{\mathrm{B}} T=20 \Gamma$, and $b<0$.

in Eq. (2). Clearly, the virtual transitions responsible for the Lamb shifts blur the otherwise polygonal Fano pattern.

At first glance one can observe a sub-Poissonian shot noise $1 / 2<F<1$ in the transport regime and both Poissonian, $F=1$, and super-Poissonian, $F>1$, shot noises in the regions of vanishing current. Furthermore, $F$ diverges when $V_{\mathrm{b}} \rightarrow 0$ due to Johnson-Nyquist noise. Finally, vertical steps in the Fano factor are clearly visible at the center of the threeand five-particle Coulomb diamonds. At these positions the energy levels of the states with one electron more or less than the participating Coulomb- or interference-blocked state are aligned, and a little change in the gate voltage favors one or the other side in transport, leading to a sudden change in the statistics that is unaffected by the bias voltage [34,35].

The complexity of the Fano pattern increases with growing electron filling, from right to left. The breaking of electronhole symmetry is strikingly revealed in a Fano factor smaller (larger) than 1 in the transport (blockade) regions involving the $N=0(6)$ and $N=1$ (5) ground states. Moreover, large values of $F$ are observed for intermediate filling. The Fano map at low filling is easily understood by observing that the oneelectron ground states $\left\{\left|1, E_{1_{0}} ; 1 / 2, \pm 1 / 2,0\right\rangle\right\}$ are only spin degenerate. Then, in the region with zero- and one-electron occupation, the Fano map resembles that of the single-impurity Anderson model, with $F=5 / 9$ and $F=1 / 2$ in the transport regions and $F=1$ at the Coulomb blockade [7]. At larger filling $N \geqslant 2$, super-Poissonian noise signals the presence of fast $(f)$ and slow $(s)$ channels. In this situation the Fano factor can be described in terms of effective filling rates $\Gamma_{\alpha}^{p}=R_{\alpha}^{p} \Gamma_{0 \alpha}$, $p=s, f$, as shown in Appendix B, as

$$
F_{n v}=1+\frac{2 \Gamma_{L}^{f}}{\Gamma_{L}^{s}+\Gamma_{R}^{s}}, \quad \mu_{L}>\mu_{R},
$$

where $\Gamma_{0 \alpha}=2 \pi|t|^{2} D_{\alpha} / \hbar$ is the bare tunneling rate for lead $\alpha$, proportional to the density of states at the Fermi energy $D_{\alpha}$. In the following we assume identical leads, such that $\Gamma_{0 L}=$ $\Gamma_{0 R}=\Gamma_{0}$ [see Fig. 1(d)]. The coefficients $R_{\alpha}^{p}$ weight the fast and slow channels and account for both spin degeneracies and orbital interference. The latter is ubiquitous in our symmetric TQD. Hence, even at Coulomb blockade, the observed values of $F$ and $F_{n v}$ cannot be simply explained in terms of the channel blockade mechanism [10]. This requires a Coulombblockaded level, and excited states in the transport window which provide a fast transport channel [see Fig. 1(b)]. The larger the excited states' degeneracy is, the larger the Fano factor is. Consider the Coulomb diamond with $N=2$ at the bias value indicated by the symbol * in Fig. 1(c), where only the ground state $\left|2_{0}\right\rangle \equiv\left|2, E_{2_{0}} ; 0,0,0\right\rangle$ and the first set of excited levels given by the sextuplet $\left\{\left|2, E_{2_{1}} ; 1, S_{z}, \pm 1\right\rangle, S_{z}=0, \pm 1\right\}$ enters the transport window. By applying Eq. (4) naively assuming that $R_{\alpha}^{p}$ is just the channel multiplicity $\left(R_{\alpha}^{s}=1\right.$ and $R_{\alpha}^{f}=6$ ), one predicts $F_{n v}=7$, which is bigger than the observed value $F_{n v} \approx 2$. At higher bias, transitions to the states $\left|2, E_{2_{2}} ; 0,0, \pm 1\right\rangle$ and $\left|3, E_{3_{0}} ; 1 / 2, \pm 1 / 2, \pm 1\right\rangle$ enter the bias window, and even larger values of $F_{n v}$ are expected. This is not observed in our TQD, where $F_{n v}<7$. However, the sheer number of open transitions makes analytics practically impossible, especially since three of these states are orbitally degenerate.

At the interference blockade, with the blocking state being decoupled at the right lead [see Fig. 1(c)], Eq. (4) holds with $\Gamma_{R}^{s}=0$. For transitions to the left lead through coupled and decoupled states one would naively expect $R_{L}^{f}=R_{L}^{s}$ and therefore a Fano factor $F_{n v}=3$, as found in many systems [9-12]. The value $F_{n v}=5 / 3$ observed, e.g., at the resonance involving the ground states $\left|2_{0}\right\rangle$ and $\left\{\left|3, E_{3_{0}} ; 1 / 2, \pm 1 / 2, \pm 1\right\rangle\right\}$ [see Fig. 2(c)] again indicates that the evaluation of the weights $R_{\alpha}^{p}$ requires precise analysis of interference with its associated "dark states". In this respect we dedicate the next section to studying the generic form of a dark state and give specific examples.

\section{DARK STATES OF A $C_{3 v}$ SYMMETRIC TQD}

When a set of orbitally degenerate levels participates in transport, interference can inhibit the escape from one manybody state with $N$ particles at one lead, such that electrons can leave this so-called dark state (DS) only via thermal activation through the other lead or via virtual excitations [see Fig. 1(c)]. This yields current suppression. In the following we focus exemplarily on transitions blocked at the right lead which involve an orbitally degenerate multiplet with $N$ particles and a singlet with $N-1$ particles. We denote the latter state, which necessarily has $L_{z}=0$, by $|N-1 ; 0\rangle$ and define a DS through the requirement $\left\langle N-1 ; 0\left|d_{1 \sigma}\right| N ; \mathrm{DS}\right\rangle=0$. Because a $L_{z}=0$ state and $d_{1 \sigma}$ are invariant upon a reflection $\sigma_{v 1}$ which leaves the site 1 invariant and sends $2 \leftrightarrow 0$, such a blocking state must 
be antisymmetric under $\sigma_{v 1}$. Expressing $d_{1 \sigma}$ in the angular momentum basis, we find for the DS the antibonding linear combination

$$
\left|N, \alpha_{i} ; \mathrm{DS}\right\rangle=\frac{1}{\sqrt{2}}\left[e^{i \frac{2 \pi}{3}}\left|N, \alpha_{i}, 1\right\rangle-e^{-i \frac{2 \pi}{3}}\left|N, \alpha_{i},-1\right\rangle\right],
$$

where $\alpha_{i}=\left\{E_{N_{i}} ; S, S_{z}\right\}$ accounts for the energy and spin of the DS. Thus a DS is an antibonding combination of states with angular momentum $L_{z}= \pm 1$. Note that this result is independent of spin degrees of freedom. Indeed, this state fulfills

$$
\begin{aligned}
& \left\langle N-1 ; 0\left|d_{1 \sigma}\right| N ; \mathrm{DS}\right\rangle \\
& =\left\langle N-1 ; 0\left|\sum_{l} e^{-i l 2 \pi / 3} d_{l \sigma}\left[e^{i 2 \pi / 3}|N ; 1\rangle-e^{-i 2 \pi / 3}|N ;-1\rangle\right]\right.\right. \\
& =\left\langle N-1 ; 0\left|d_{l=1 \sigma}\right| N ; 1\right\rangle-\left\langle N-1 ; 0\left|d_{l=-1 \sigma}\right| N ;-1\right\rangle \\
& =0
\end{aligned}
$$

which shows that a transition is forbidden at the right lead. The bonding linear combination corresponds to the coupled state $|N ; \mathrm{CS}\rangle$. Expressing the dark states in position basis $\{0 \uparrow, 1 \uparrow, 2 \uparrow ; 0 \downarrow, 1 \downarrow, 2 \downarrow\}$ yields further insight into the blocking mechanism. Let us consider their composition for increasing electron filling. The dark state for the one-particle first excited state with $S_{z}=1 / 2$ is

$$
\left|1, E_{1} ; \frac{1}{2}, \frac{1}{2} ; \mathrm{DS}\right\rangle=\frac{1}{\sqrt{2}}\left(\infty_{\infty}-\infty \infty\right),
$$

and similarly for the dark state with $S_{z}=-1 / 2$. Thus we recover the familiar result by Hsieh et al. [30], where the
DS is a state without occupation of the right-coupled dot 1. On the other hand, the vanishing of the matrix element in Eq. (6) also comes naturally from the fact that the DS (7) is antisymmetric under the operation $\sigma_{v 1}$ while both $d_{1 \sigma}$ and the vacuum state $\left|0, \alpha_{0}, 0\right\rangle$ are symmetric.

For the two-particle first excited state with $S_{z}=1$ we obtain

$$
\left|2, \alpha_{1} ; \mathrm{DS}\right\rangle=\frac{1}{\sqrt{6}}\left(\phi_{\infty}+\sigma_{\phi} \phi+2 \phi_{\infty}\right),
$$

and similarly for $S_{z}=-1$. For the case $S_{z}=0$ we find

$$
\begin{aligned}
& \left|2, E_{2_{1}} ; 1,0 ; \mathrm{DS}\right\rangle=\frac{1}{2 \sqrt{3}}[(\$ \phi-\infty \phi) \\
& -(\underset{\phi}{\phi}-\infty \phi) \\
& \left.+2\left(\phi_{\infty}^{\infty}-\phi_{\infty}\right)\right]
\end{aligned}
$$

The composition of states shown in Eqs. (8) and (9) is counterintuitive because they admit finite occupation of dot 1 . However, again, the vanishing of the transition amplitude (6) results from the fact that the DS is antisymmetric with respect to the reflection $\sigma_{v 1}$ and the state $\left|1, \alpha_{0}, 0\right\rangle$ is symmetric. Notice that, crucially, the two contributions with single occupation of dot 1 give a contribution of opposite sign to the amplitude (6).

The three-particle ground state with $S_{z}=1 / 2$ is given by the intricate superposition



$$
\begin{aligned}
& +\left(v_{0,1}+v_{0,0}+v_{0,-1}\right)\left(\boldsymbol{\phi}_{\phi}^{\phi}+\phi^{\phi}\right)+\left(v_{0,1}+v_{0,0}-2 v_{0,-1}\right)\left(\phi^{\phi}+\underset{\phi}{\phi}\right) \\
& \left.+2\left(v_{0,1}+v_{0,0}+v_{0,-1}\right) \underset{\phi}{\phi}\right] \text {, }
\end{aligned}
$$

where $v_{x, y}$ is given in the caption of Table I. Analogously the state with $S_{z}=-1 / 2$ can be constructed. Again, the vanishing of the transition amplitude (6) results from a nontrivial quantum cancellation.

\section{INTERFERENCE BLOCKADE AT THE $2_{0} \leftrightarrow 3_{0}$ RESONANCE}

We apply the results above to investigate the bias region involving transitions among an $N$-particle ground-state singlet and an orbitally degenerate $(N+1)$-particle ground state. We exemplarily choose the $2_{0} \leftrightarrow 3_{0}$ resonance where, as seen in Fig. 2(c), $F_{n v}=5 / 3$, but the results apply to other resonances as well. To this extent, let us observe that since the total Hamiltonian $H$ conserves particles, energy, and spin, the stationary density matrix $\rho^{\infty}$ has a block diagonal structure, with blocks $\rho^{N S S_{z}}(E)$ of definite $N, S, S_{z}$, and $E$ [36]. Due to the equivalence of the configurations with different $S_{z}$ for the dynamics, we introduce the matrices $\rho_{L_{z} L_{z}^{\prime}}^{N}(E):=$ $\sum_{S_{z}}\left[\rho^{N S S_{z}}(E)\right]_{L_{z} L_{z}^{\prime}}$. For example, since there exists only one configuration for the $N=2$ ground state, $\rho^{2}$ is a number. On the other hand, $\rho^{3}\left(E_{3_{0}}\right)$ is the $2 \times 2$ matrix associated with the three-particle ground-state quadruplet. By using the Wigner-Eckart theorem [37] to calculate matrix elements of the operators, $d_{\alpha \sigma}^{\dagger}$ and $d_{\alpha \sigma}$, between states of different particle numbers and spins and summing over $\sigma$, Eq. (2) yields for the case of unidirectional transport near the $2_{0} \leftrightarrow 3_{0}$ resonance

$$
0=-\frac{i}{\hbar}\left[H_{\mathrm{LS}}, \rho^{3}\right]+2 \Gamma \mathcal{R}_{L} \rho^{2}-\frac{\Gamma}{2}\left\{\mathcal{R}_{R}, \rho^{3}\right\},
$$

which, together with $\operatorname{Tr}_{\mathrm{TQD}}\{\rho\}=1$, fully determines $\rho^{2}\left(E_{2_{0}}\right)$ and $\rho^{3}\left(E_{3_{0}}\right)$. The Lamb shift Hamiltonian can be cast, follow- 
ing Donarini et al. [18], into the form $H_{\mathrm{LS}}=\hbar \sum_{\alpha} \omega_{\alpha} \mathcal{R}_{\alpha}$. The precession frequencies $\omega_{\alpha}$ account for virtual transitions from the three-particle ground states to the states with two and four particles and are independent of $S_{z}$. We find

$$
\begin{aligned}
\omega_{\alpha}= & \frac{\Gamma_{0}}{2 \pi} \sum_{\tau, E} p_{\alpha}\left(E-E_{3_{0}}\right) \\
& \times\left\langle 3, E_{3_{0}} ; \frac{1}{2}, S_{z}, L_{z}\left|d_{0 \tau} \mathcal{P}_{4, E} d_{0 \tau}^{\dagger}\right| 3, E_{3_{0}} ; \frac{1}{2}, S_{z},-L_{z}\right\rangle \\
& +p_{\alpha}\left(E_{3_{0}}-E\right) \\
& \times\left\langle 3, E_{3_{0}} ; \frac{1}{2}, S_{z}, L_{z}\left|d_{0 \tau}^{\dagger} \mathcal{P}_{2, E} d_{0 \tau}\right| 3, E_{3_{0}} ; \frac{1}{2}, S_{z},-L_{z}\right\rangle,
\end{aligned}
$$

where $\mathcal{P}_{N E}=\sum_{S_{z}, L_{z}}\left|N, E ; S, S_{z}, L_{z}\right\rangle\left\langle N, E ; S, S_{z}, L_{z}\right|$ is the projector on the $N$-particle level with energy $E$ and spin $S$. We defined the function $p_{\alpha}(\Delta E)=$ $-\operatorname{Re} \psi\left[1 / 2+i\left(\Delta E-\mu_{\alpha}\right) /\left(2 \pi k_{\mathrm{B}} T\right)\right]$, where $T$ is the temperature, $\psi$ is the digamma function, and $\mu_{\alpha}$ is the chemical potential of lead $\alpha$. The matrices $\mathcal{R}_{\alpha}$ have in the angular momentum basis the form $\left(\mathcal{R}_{\alpha}\right)_{\ell \ell^{\prime}}=e^{i \alpha\left(\ell-\ell^{\prime}\right) 2 \pi / 3}, \ell, \ell^{\prime}= \pm 1$. We defined $\Gamma=a \Gamma_{0}$, where $a=\frac{1}{2} \sum_{\sigma}\left|\left\langle 3, E_{3_{0}} ; \frac{1}{2},-\sigma, 1\left|d_{\alpha \sigma}^{\dagger}\right| 2_{0}\right\rangle\right|^{2}$. Notice that one cannot diagonalize $\mathcal{R}_{L}$ and $\mathcal{R}_{R}$ simultaneously. In the basis spanned by $|3, \mathrm{DS}\rangle$ and $|3, \mathrm{CS}\rangle$ we get

$$
\mathcal{R}_{R}=\left(\begin{array}{ll}
0 & 0 \\
0 & 2
\end{array}\right), \quad \mathcal{R}_{L}=\frac{1}{2}\left(\begin{array}{cc}
3 & -i \sqrt{3} \\
i \sqrt{3} & 1
\end{array}\right),
$$

and $\rho^{3}\left(E_{3_{0}}\right)=p(I+\mathbf{n} \cdot \boldsymbol{\sigma}) / 2$, where $\boldsymbol{n}$ is the Bloch vector corresponding to the orbitally degenerate state, $\sigma$ is the vector of Pauli matrices, $p=\rho_{d d}+\rho_{c c}$, and the decoupled state points along the $z$ axis. Neglecting the Lamb shift term, the matrix $\rho^{3}\left(E_{3_{0}}\right)$ is diagonal, with elements $\rho^{d d}=1, \rho^{c c}=0$ at the deep interference blockade. The diagonal elements 0,2 and $3 / 2,1 / 2$ of $\mathcal{R}_{\alpha}$ correspond to the weights $R_{R}^{s}, R_{R}^{f}$ and $R_{L}^{s}$, $R_{L}^{f}$, respectively, entering Eq. (4). Notice that this yields the counterintuitive result $R_{L}^{f} \neq R_{L}^{s}$. Using these values, we find $F_{n v}=5 / 3$.

So far the effect of the Lamb shift Hamiltonian $H_{L S}$ has been neglected. An analytical treatment of the precessional dynamics is possible in the parameter region involving the $N=5$ and $N=6$ ground states, as discussed below.

\section{INTERFERENCE BLOCKADE AT THE $5_{0} \leftrightarrow 6_{0}$ RESONANCE}

The Lamb shift term describes a precession of the Bloch vector $\boldsymbol{n}$ around an axis set by the matrices $\mathcal{R}_{\alpha}$. The populations of the coupled and dark states are thus affected by partially coherent gain and loss, and the blockade is perfect only when $\omega_{L}=0$. We choose the $5_{0} \leftrightarrow 6_{0}$ resonance at $\xi=-7.5|b|$, indicated by a green dashed line in Fig. 2, to study the effect of this precession.

\section{A. Hole transport}

The dynamics between the $N=5$ and $N=6$ ground states is easily described in terms of hole transport. Then, Eqs. (4), (11), and (13) apply upon exchange of $L \leftrightarrow R$ together with



FIG. 3. Bias trace of (a) current, (b) Fano factor, and (c) precession frequencies $\omega_{L, R}$ at $\xi=-7.5|b|$, corresponding to the green dashed line in Fig. 2. The numerical data agree well with analytical expressions from the text. Inset: The population of the coupled (CS) and dark (DS) states is affected by effective filling rates and by a precessional dynamics with frequency $\omega_{R}$.

$3 \rightarrow 5,2 \rightarrow 6$, which yields

$$
\begin{aligned}
& 0=-\frac{i}{\hbar}\left[H_{\mathrm{LS}}, \rho^{5}\right]+2 \Gamma \mathcal{R}_{R} \rho^{6}-\frac{\Gamma}{2}\left\{\mathcal{R}_{L}, \rho^{5}\right\}, \\
& 0=\Gamma \operatorname{Tr}_{\mathrm{TQD}}\left(\mathcal{R}_{L} \rho^{5}\right)-4 \Gamma \rho^{6},
\end{aligned}
$$

where we use $a=\frac{1}{2} \sum_{\sigma}\left|\left\langle 5, E_{5_{0}} ; \frac{1}{2},-\sigma, 1\left|d_{\alpha \sigma}\right| 6\right\rangle\right|^{2}=\frac{1}{3}$ and $\Gamma=a \Gamma_{0}$.

Bias traces of currents and Fano factors at the gate voltage corresponding to $\xi=-7.5|b|$ are shown in Figs. 3(a) and 3(b). The current $I_{n v}$, which does not account for the Lamb shifts, is exponentially suppressed in the voltage range $0.5<e V_{\mathrm{b}} /|b|<$ 3.5. The associated Fano factor takes the values $F_{n v}=5 / 3$ at low bias and $F_{n v}=4 / 3$ above $e V_{\mathrm{b}} /|b|=2$ when transitions from $5_{0}$ to the four-particle ground states $\left\{\left|4, E_{4_{0}} ; 1, S_{z}, 0\right\rangle\right\}$ dominate the bottleneck process for transport, as shown in Appendix D. Virtual transitions modify this picture: the current $I$ (Fano factor $F$ ) varies with bias voltage and has a minimum (maximum) at $\omega_{R} \approx 0$. In the following we investigate how the Lamb shift Hamiltonian affects the dark state of the fiveparticle ground states and the resulting Fano factor.

\section{B. Interference dynamics}

The precession frequencies $\omega_{\alpha}$ from Eq. (12) account for virtual transitions from the five-particle ground states $\left\{\left|5, E_{5_{0}} ; 1 / 2, \pm 1 / 2, \pm 1\right\rangle\right\}$ to the state $\left|6_{0}\right\rangle \equiv\left|6, E_{6} ; 0,0,0\right\rangle$ and to levels with energies $E_{4_{0,1,2,3}}$. Their bias dependence is shown in Fig. 3(c). The stationary density matrix in the 



FIG. 4. (a) Current and (b) Fano factor as a function of detuning at $V_{\mathrm{g}}=-7.5|b|$ for different bias voltages. In the limit of large detuning where $\Delta E \gg \hbar \Gamma_{0}$, the current is $I=-e 4 \Gamma_{0} / 15$ and the Fano factor $F=17 / 25$. For vanishing detuning $F=5 / 3$ is recovered at full blockade $\left(e V_{\mathrm{b}} \approx 1.15|b|\right)$.

ordering $\rho^{d d}, \rho^{c c}, \rho^{6}, \rho^{d c}, \rho^{c d}$ obtained as a solution to Eq. (14) is

$$
\rho^{\infty}=\frac{1}{D}\left(\begin{array}{c}
D-3 \omega_{R}^{2} \\
2 \omega_{R}^{2} \\
\omega_{R}^{2} \\
-\sqrt{3} \omega_{R}\left[\Gamma-i 2\left(\omega_{L}-\omega_{R}\right)\right] \\
-\sqrt{3} \omega_{R}\left[\Gamma+i 2\left(\omega_{L}-\omega_{R}\right)\right]
\end{array}\right)
$$

with $D=2 \Gamma^{2}+8 \omega_{L}^{2}-12 \omega_{L} \omega_{R}+9 \omega_{R}^{2}$. The corresponding current is $I=-e 4 \Gamma \omega_{R}^{2} / 3 D$. For $\omega_{R} \rightarrow 0$ the system gets quadratically stuck in the decoupled state, and thus current is suppressed. The resulting Fano factor is

$$
\begin{aligned}
F= & \frac{16 \omega_{R}^{2}\left(2 \Gamma^{2}+53 \omega_{L}^{2}\right)-176 \omega_{L} \omega_{R}\left(\Gamma^{2}+4 \omega_{L}^{2}\right)}{3 D^{2}} \\
& +\frac{20\left(\Gamma^{2}+4 \omega_{L}^{2}\right)^{2}-576 \omega_{L} \omega_{R}^{3}+195 \omega_{R}^{4}}{3 D^{2}}
\end{aligned}
$$

which to lowest order in $\omega_{R}$ is $F=5 / 3+16 \omega_{L} \omega_{R} /$ $\left(\Gamma^{2}+4 \omega_{L}^{2}\right)$. Therefore the limit of $F=5 / 3$ is recovered at the complete blockade. Since $\omega_{L}>0$, the Fano factor is not maximal at $\omega_{R}=0$ but instead at a little lower bias voltage.

\section{Robustness against perturbations}

To check the influence of a weak perturbation which lifts degeneracies, we restrict ourselves again to the $5_{0} \leftrightarrow 6$ resonance. Then this perturbation changes the on-site energies of the orbitally degenerate $\left|5_{0}\right\rangle$ states and is given by a Hamiltonian $H_{\Delta}=-\Delta E \sigma_{z} / 2$, which, rotated to the coupled and decoupled basis, takes the form

$$
H_{\Delta}=\frac{1}{2}\left(\begin{array}{cc}
0 & \Delta E \\
\Delta E & 0
\end{array}\right) .
$$

If one assumes weak coupling to the leads, $\Gamma \ll k_{B} T$, and also that $\Delta E \ll k_{B} T$, the dissipative part of the unidirectional master equation is unaffected by the changes. Therefore the master equation (14) holds with the substitution $H_{\mathrm{LS}} \rightarrow$ $H_{\mathrm{LS}}+H_{\Delta}$. The resulting current and Fano factor at the $5_{0} \leftrightarrow 6$ resonance are shown as a function of the detuning in Fig. 4.
The results of this paper are robust for a perturbation strength up to the order of $\Delta E \lesssim 0.01 \hbar \Gamma_{0}$. In the limit of large detuning where $k_{B} T \gg \Delta E \gg \hbar \Gamma_{0}$ the current is $I=-e 4 \Gamma_{0} / 15$, and the Fano factor $F=17 / 25$. This Fano factor can be explained by the one for a single resonant level $F=\left(R_{L}^{2}+R_{R}^{2}\right) /\left(R_{L}+R_{R}\right)^{2}$, where, due to the fourfold degeneracy of $\left|5_{0}\right\rangle, R_{L}=4 R_{R}$. Notice that $F=5 / 9$ on the right side of the stability diagram [7] in Fig. 2(c) can be obtained with $R_{L}=2 R_{R}$.

\section{CONCLUSIONS}

Using a full counting statistics approach in Liouville space, we obtained the Fano stability diagram of a $C_{3 v}$ symmetric TQD. In the region of current suppression the Fano factor helps unravel the underlying blocking mechanisms. Poissonian statistics suggests "classical" Coulomb blockade, whereas super-Poissonian noise points to the presence of fast and slow channels, the latter including dark states. A population redistribution between dark and coupled states, induced by virtual excitations, results in a nontrivial bias dependence of the Fano factor. The value attained by the Fano factor at specific gate and bias voltages further reveals the internal structure of the dark states.

This work focused on the impact of the $C_{3 v}$ symmetry on noise, and thus restriction to sequential tunneling already yields interesting results. However, cotunneling contributions $[27,28]$ might additionally influence the noise features and should be further investigated for a $C_{3 v}$ symmetric setup.

\section{ACKNOWLEDGMENT}

The authors acknowledge financial support from the Deutsche Forschungsgemeinschaft via GRK 1570 and SFB 689 .

\section{APPENDIX A: MANY-BODY SPECTRUM AND EIGENFUNCTIONS OF A SYMMETRIC TQD}

All eigenvectors and the associated eigenvalues in the occupation number basis of the TQD Hamiltonian equation (1) are reported in Tables I and II. We also included all quantum numbers necessary to classify these states in terms of total particle number $N$, energy $E$, total spin $S^{2}$, and projections 
TABLE I. Eigenvalues and eigenstates of a $C_{3 v}$ symmetric TQD Hamiltonian for occupation numbers $N=0-3$. Such eigenvectors are furthermore characterized by the spin quantum numbers $S$ and $S_{z}$ and by the orbital quantum number $L_{z}$. Their composition in the basis of the occupation number vectors is provided in the rightmost column. The ordering of the eigenergies depends on the TQD parameters $b, U$ and $V$. We choose $U=5|b|, V=2|b|$, and $b<0$. We define $a=(U-V) /(9 b), \theta=\arccos \left\{\left[\left(3 a^{2}\right) /\left(1+3 a^{2}\right)\right]^{3 / 2}\right\} / 3, \lambda_{\alpha}=2 \sqrt{\left(1+a^{2}\right) / 3} \cos \left(\theta+\alpha \frac{2 \pi}{3}\right)$, $v_{x, y}=\left(a-\lambda_{x}\right)\left|\left(a-\lambda_{x}\right)^{2}-1\right| /\left(a-\lambda_{x}-y\right) \sqrt{3\left(a-\lambda_{x}\right)^{4}+1}, s_{x}=\sqrt{9 x^{2} b^{2}+x b / 2(U-V)+(U-V)^{2}}$, and $\phi_{x}=\frac{1}{2} \arctan \left(\frac{2 \sqrt{2}(U-V)}{U-V+9 x b}\right)$.

\begin{tabular}{|c|c|c|c|c|c|}
\hline$N$ & Eigenenergy & $S$ & $S_{z}$ & $L_{z}$ & Eigenstate in the basis $\left\{\left|n_{0 \uparrow}, n_{1 \uparrow}, n_{-1 \uparrow} ; n_{0 \downarrow}, n_{1 \downarrow}, n_{-1 \downarrow}\right\rangle\right\}$ \\
\hline 0 & $E_{0}=0$ & 0 & 0 & 0 & $|000,000\rangle$ \\
\hline \multirow{6}{*}{1} & \multirow{2}{*}{$E_{1_{0}}=\xi-\frac{U}{2}-2 V+2 b$} & \multirow{2}{*}{$\frac{1}{2}$} & \multirow{2}{*}{\begin{tabular}{|c|}
$-\frac{1}{2}$ \\
$\frac{1}{2}$ \\
\end{tabular}} & \multirow{2}{*}{0} & $|000,100\rangle$ \\
\hline & & & & & $|100,000\rangle$ \\
\hline & \multirow{4}{*}{$E_{1_{1}}=\xi-\frac{U}{2}-2 V-b$} & \multirow{4}{*}{$\frac{1}{2}$} & \multirow{2}{*}{$-\frac{1}{2}$} & -1 & $|000,001\rangle$ \\
\hline & & & & 1 & $|000,010\rangle$ \\
\hline & & & \multirow{2}{*}{$\frac{1}{2}$} & -1 & $|001,000\rangle$ \\
\hline & & & & 1 & $|010,000\rangle$ \\
\hline \multirow{14}{*}{2} & $E_{2_{0}}=2 \xi-U-3 V+b+\frac{U-V}{2}-s_{-2}$ & 0 & 0 & 0 & $\cos \left(\phi_{-2}\right)|100,100\rangle-\sin \left(\phi_{-2}\right) \frac{1}{\sqrt{2}}(|010,001\rangle+|001,010\rangle)$ \\
\hline & \multirow{6}{*}{$E_{2_{1}}=2 \xi-U-3 V+b$} & \multirow{6}{*}{1} & \multirow{2}{*}{-1} & -1 & $|000,101\rangle$ \\
\hline & & & & 1 & $|000,110\rangle$ \\
\hline & & & \multirow{2}{*}{0} & -1 & $\frac{1}{\sqrt{2}}(|100,001\rangle-|001,100\rangle)$ \\
\hline & & & & 1 & $\frac{1}{\sqrt{2}}(|100,010\rangle-|010,100\rangle)$ \\
\hline & & & \multirow{2}{*}{1} & -1 & $|101,000\rangle$ \\
\hline & & & & 1 & $|110,000\rangle$ \\
\hline & \multirow{2}{*}{$E_{2_{2}}=2 \xi-U-3 V-\frac{b}{2}+\frac{U-V}{2}-s_{1}$} & \multirow{2}{*}{0} & \multirow[t]{2}{*}{0} & -1 & $\cos \left(\phi_{1}\right)|010,010\rangle-\sin \left(\phi_{1}\right) \frac{1}{\sqrt{2}}(|100,001\rangle+|001,100\rangle)$ \\
\hline & & & & 1 & $\cos \left(\phi_{1}\right)|001,001\rangle-\sin \left(\phi_{1}\right) \frac{1}{\sqrt{2}}(|100,010\rangle+|010,100\rangle)$ \\
\hline & \multirow{3}{*}{$E_{2_{3}}=2 \xi-U-3 V-2 b$} & \multirow{3}{*}{1} & -1 & \multirow{3}{*}{0} & $|000,011\rangle$ \\
\hline & & & 0 & & $\frac{1}{\sqrt{2}}(|010,001\rangle-|001,010\rangle)$ \\
\hline & & & 1 & & $|011,000\rangle$ \\
\hline & \multirow{2}{*}{$E_{2_{4}}=2 \xi-U-3 V-\frac{b}{2}+\frac{U-V}{2}+s_{1}$} & \multirow[t]{2}{*}{0} & \multirow[t]{2}{*}{0} & -1 & $\sin \left(\phi_{1}\right)|010,010\rangle+\cos \left(\phi_{1}\right) \frac{1}{\sqrt{2}}(|100,001\rangle+|001,100\rangle)$ \\
\hline & & & & 1 & $\sin \left(\phi_{1}\right)|001,001\rangle+\cos \left(\phi_{1}\right) \frac{1}{\sqrt{2}}(|100,010\rangle+|010,100\rangle)$ \\
\hline & $E_{2_{5}}=2 \xi+b-U-3 V+\frac{U-V}{2}+s_{-2}$ & 0 & 0 & 0 & $\sin \left(\phi_{-2}\right)|100,100\rangle+\cos \left(\phi_{-2}\right) \frac{1}{\sqrt{2}}(|010,001\rangle+|001,010\rangle)$ \\
\hline \multirow{20}{*}{3} & & & $-\frac{1}{2}$ & -1 & $\left.\left|v_{0,1}\right| 100,101\right\rangle-v_{0,0}|010,110\rangle-v_{0,-1}|001,011\rangle$ \\
\hline & $E_{3_{0}}=3 \xi-\frac{3}{2} U-3 V+\frac{2}{3}(U-V)\left[1-\lambda_{0} /(2|a|)\right]$ & $\frac{1}{2}$ & $-\overline{2}$ & 1 & $v_{0,1}|100,110\rangle+v_{0,0}|001,101\rangle-v_{0,-1}|010,011\rangle$ \\
\hline & & & $\frac{1}{a}$ & -1 & $v_{0,1}|101,100\rangle-v_{0,0}|110,010\rangle-v_{0,-1}|011,001\rangle$ \\
\hline & & & 2 & 1 & $\left.\left|v_{0,1}\right| 110,100\right\rangle-v_{0,0}|101,001\rangle+v_{0,-1}|011,010\rangle$ \\
\hline & & & $-\frac{3}{2}$ & & $|000,111\rangle$ \\
\hline & $E_{3_{1}}=3 \xi-\frac{3}{2} U-3 V$ & $\frac{3}{2}$ & $-\frac{1}{2}$ & 0 & $\frac{1}{\sqrt{3}}(|001,110\rangle-|010,101\rangle+|100,011\rangle)$ \\
\hline & & & $\frac{1}{2}$ & & $\frac{1}{\sqrt{3}}(|011,100\rangle-|101,010\rangle+|110,001\rangle)$ \\
\hline & & & $\frac{3}{2}$ & & $|111,000\rangle$ \\
\hline & & & $-\frac{1}{2}$ & -1 & $v_{1,1}|110,100\rangle-v_{1,0}|101,001\rangle+v_{1,-1}|011,010\rangle$ \\
\hline & $E_{3_{2}}=3 \xi-\frac{3}{2} U-3 V+\frac{2}{3}(U-V)\left[1-\lambda_{1} /(2|a|)\right]$ & $\frac{1}{2}$ & $\overline{2}$ & 1 & $v_{1,1}|100,110\rangle+v_{1,0}|001,101\rangle-v_{1,-1}|010,011\rangle$ \\
\hline & & & $\underline{1}$ & -1 & $v_{1,1}|101,100\rangle-v_{1,0}|110,010\rangle-v_{1,-1}|011,001\rangle$ \\
\hline & & & $\overline{2}$ & 1 & $v_{1,1}|100,101\rangle-v_{1,0}|010,110\rangle-v_{1,-1}|001,011\rangle$ \\
\hline & & & $-\frac{1}{2}$ & & $\frac{1}{\sqrt{2}}(|001,110\rangle-|100,011\rangle)$ \\
\hline & $E_{3_{3}}=3 \xi-\frac{3}{2} U-3 V+(U-V)$ & $\frac{1}{2}$ & 2 & 0 & $\frac{1}{\sqrt{6}}(|001,110\rangle+2|010,101\rangle+|100,011\rangle)$ \\
\hline & & & $\frac{1}{a}$ & & $\frac{1}{\sqrt{6}}(|110,001\rangle+2|101,010\rangle+|011,100\rangle)$ \\
\hline & & & $\overline{2}$ & & $\frac{1}{\sqrt{2}}(|110,001\rangle-|011,100\rangle)$ \\
\hline & & & $-\frac{1}{2}$ & -1 & $\left.\left|v_{-1,1}\right| 100,101\right\rangle-v_{-1,0}|010,110\rangle-v_{-1,-1}|001,011\rangle$ \\
\hline & $E_{3_{4}}=3 \xi-\frac{3}{2} U-3 V+\frac{2}{3}(U-V)\left[1-\lambda_{-1} /(2|a|)\right]$ & $\frac{1}{2}$ & $-\overline{2}$ & 1 & $\left.\left|v_{-1,1}\right| 100,110\right\rangle+v_{-1,0}|001,101\rangle-v_{-1,-1}|010,011\rangle$ \\
\hline & & & $\frac{1}{1}$ & -1 & $\left.\left|v_{-1,1}\right| 101,100\right\rangle-v_{-1,0}|110,010\rangle-v_{-1,-1}|011,001\rangle$ \\
\hline & & & 2 & 1 & $\left.\left|v_{-1,1}\right| 110,100\right\rangle-v_{-1,0}|101,001\rangle+v_{-1,-1}|011,010\rangle$ \\
\hline
\end{tabular}


TABLE II. Eigenvalues and eigenstates of a $C_{3 v}$ symmetric TQD for electron numbers $N=4-6$. The parameters and notations are the same as in Table I. The ordering is for $U=5|b|, V=2|b|$, and $b<0$. We define $s_{x}=\sqrt{9 x^{2} b^{2}+x b / 2(U-V)+(U-V)^{2}}$ and $\phi_{x}=$ $\frac{1}{2} \arctan \left(\frac{2 \sqrt{2}(U-V)}{U-V+9 x b}\right)$.

\begin{tabular}{|c|c|c|c|c|c|}
\hline$N$ & Eigenenergy & $S$ & $S_{z}$ & $L_{z}$ & Eigenstate in the basis $\left\{\left|n_{0 \uparrow}, n_{1 \uparrow}, n_{-1 \uparrow} ; n_{0 \downarrow}, n_{1 \downarrow}, n_{-1 \downarrow}\right\rangle\right\}$ \\
\hline & \multirow{3}{*}{$E_{4_{0}}=4 \xi+2 b-U-3 V$} & \multirow{3}{*}{1} & -1 & \multirow{3}{*}{0} & $|100,111\rangle$ \\
\hline & & & 0 & & $\frac{1}{\sqrt{2}}(|101,110\rangle-|110,101\rangle)$ \\
\hline & & & 1 & & $|111,100\rangle$ \\
\hline & \multirow{2}{*}{$E_{4_{1}}=4 \xi-U-3 V+\frac{b}{2}+\frac{U-V}{2}-s_{-1}$} & \multirow{2}{*}{0} & \multirow{2}{*}{0} & -1 & $\cos \left(\phi_{-1}\right)|110,110\rangle+\sin \left(\phi_{-1}\right) \frac{1}{\sqrt{2}}(|011,101\rangle+|101,011\rangle)$ \\
\hline & & & & 1 & $\cos \left(\phi_{-1}\right)|101,101\rangle-\sin \left(\phi_{-1}\right) \frac{1}{\sqrt{2}}(|011,110\rangle+|110,011\rangle)$ \\
\hline \multirow{7}{*}{\multicolumn{2}{|c|}{$E_{4_{2}}=4 \xi-U-3 V-b+\frac{U-V}{2}-s_{2}$}} & 0 & 0 & 0 & $\cos \left(\phi_{2}\right)|011,011\rangle+\sin \left(\phi_{2}\right) \frac{1}{\sqrt{2}}(|101,110\rangle+|110,101\rangle)$ \\
\hline & & \multirow{2}{*}{\multicolumn{2}{|c|}{-}} & -1 & $|001,111\rangle$ \\
\hline & & & & 1 & $|010,111\rangle$ \\
\hline & & \multirow[t]{4}{*}{1} & \multirow{2}{*}{0} & -1 & $\frac{1}{\sqrt{2}}(|011,101\rangle-|101,011\rangle)$ \\
\hline & & & & 1 & $\frac{1}{\sqrt{2}}(|011,110\rangle-|110,011\rangle)$ \\
\hline & & & \multirow{2}{*}{1} & -1 & $|111,001\rangle$ \\
\hline & & & & 1 & $|111,010\rangle$ \\
\hline & \multirow{2}{*}{$E_{4_{4}}=4 \xi-U-3 V+\frac{b}{2}+\frac{U-V}{2}+s_{-1}$} & \multirow{2}{*}{0} & \multirow{2}{*}{0} & -1 & $\sin \left(\phi_{-1}\right)|110,110\rangle-\cos \left(\phi_{-1}\right) \frac{1}{\sqrt{2}}(|011,101\rangle+|101,011\rangle)$ \\
\hline & & & & 1 & $\sin \left(\phi_{-1}\right)|101,101\rangle+\cos \left(\phi_{-1}\right) \frac{1}{\sqrt{2}}(|011,110\rangle+|110,011\rangle)$ \\
\hline & $E_{4_{5}}=4 \xi-U-3 V-b+\frac{U-V}{2}+s_{2}$ & 0 & 0 & 0 & $-\sin \left(\phi_{2}\right)|011,011\rangle+\cos \left(\phi_{2}\right) \frac{1}{\sqrt{2}}(|101,110\rangle+|110,101\rangle)$ \\
\hline \multirow{6}{*}{5} & \multirow{4}{*}{$E_{5_{0}}=5 \xi-\frac{U}{2}-2 V+b$} & \multirow{4}{*}{$\frac{1}{2}$} & \multirow{2}{*}{$-\frac{1}{2}$} & -1 & $|101,111\rangle$ \\
\hline & & & & 1 & $|110,111\rangle$ \\
\hline & & & \multirow{2}{*}{$\frac{1}{2}$} & -1 & $|111,101\rangle$ \\
\hline & & & & 1 & $|111,110\rangle$ \\
\hline & \multirow{2}{*}{$E_{5_{1}}=5 \xi-\frac{U}{2}-2 V-2 b$} & \multirow{2}{*}{$\frac{1}{2}$} & \multirow{2}{*}{\begin{tabular}{|c|}
$\frac{1}{2}$ \\
$\frac{1}{2}$
\end{tabular}} & \multirow{2}{*}{0} & $|011,111\rangle$ \\
\hline & & & & & $|111,011\rangle$ \\
\hline 6 & $E_{6}=6 \xi$ & 0 & 0 & 0 & $|111,111\rangle$ \\
\hline
\end{tabular}

$S_{z}$ and $L_{z}$ of the total spin and angular momentum. The corresponding many-body state is denoted by $\left|N, E ; S, S_{z}, L_{z}\right\rangle$.

We notice that a classification of many-body states using the angular momentum quantum number $L_{z}$ has been proposed by Kostyrko and Bułka, [17] for the case of a symmetric triangular dot with only intrasite repulsion (i.e., $U \neq 0$, $V=0)$. Our analysis with $U \neq 0, V \neq 0$ thus generalizes that work and recovers the results reported by Korkusinski et al. [16], where a localized representation is used to discuss topological Hund rules and derive effective low-energy spin Hamiltonians. For finite on-site and intersite interactions $U$ and $V$, the composition of the eigenstates is the result of a complex interplay between Pauli statistics and Coulomb repulsion, and we refer to the review by Hsieh et al. [30] for useful insights. For example, for double occupancy of the TQD, the configurations with $S=1$ correspond to excited states with singly occupied dots due to the Pauli principle. The configurations with $S=0$, however, contain both doubly occupied and singly occupied dots, with weight determined by the difference $U-V$. For $U=V$, the ground state is, in the occupation number representation, the singlet $|100,100\rangle$, with equal weights on single and doubly occupied sites, as seen in Table I. The splitting between the sextuplet of excited states and the ground-state singlet is dominated by the hopping energy with a correction given by superexchange processes due to the doubly occupied singlet configurations [16]. For a TQD with occupancy $N=4$ (i.e., with two holes), the ground state is always a triplet if $b<0$, as in our work. Finally, of relevance for the discussion in the main text, the three-particle and five-particle ground states are a quadruplet due to orbital and spin degeneracies, while the associated first excited states are only spin degenerate.

\section{APPENDIX B: CURRENT AND FANO FACTOR FOR A MINIMAL MODEL WITH SLOW AND FAST CHANNELS}

Let us consider a minimal system consisting of slow and fast channels, which, for example, can be a ground state in the Coulomb blockade (CB) plus an excited state in the bias window or the coupled and decoupled states in the case of the interference blockade (IB), as depicted in Figs. 1(b) and 1(c), respectively. This system spends most of the time in the state corresponding to the slow channel, and therefore the exponentially suppressed current is dominated by the 
bottleneck process of escaping this state. The Liouvillian $\mathcal{L}$ and the tunneling Liouvillian $\mathcal{L}_{t}$ are superoperators whose matrix elements are obtained from their action on the reduced density matrix. In order to obtain a suitable representation of such Liouvillians, it is convenient to work in the Liouville space, where the density matrix elements are ordered in a vector. Let us consider a situation of positive electrochemical potential, such that particle transport occurs from the left, $L$, to the right, $R$, lead. Then, far from resonance (e.g., inside the Coulomb-blockade or interference-blockade regions), we can approximate the Fermi functions of the fast channel to 1 or 0. In a generic situation where a state $|p\rangle$ has one less electron than the fast $|f\rangle$ and slow $|s\rangle$ states, the Liouvillian in the basis $\{|p\rangle\langle p|| f\rangle,\langle f|| s\rangle,\langle s|\}$ takes the form

$$
\begin{aligned}
\mathcal{L}_{t}= & \left(\begin{array}{ccc}
-\Gamma_{L}^{f} & \Gamma_{R}^{f} & 0 \\
\Gamma_{L}^{f} & -\Gamma_{R}^{f} & 0 \\
0 & 0 & 0
\end{array}\right) \\
& +\sum_{\alpha}\left(\begin{array}{ccc}
-\Gamma_{\alpha}^{s} f_{\alpha}^{+} & 0 & \Gamma_{\alpha}^{s} f_{\alpha}^{-} \\
0 & 0 & 0 \\
\Gamma_{\alpha}^{s} f_{\alpha}^{+} & 0 & -\Gamma_{\alpha}^{s} f_{\alpha}^{-}
\end{array}\right) .
\end{aligned}
$$

The corresponding current superoperators for the right lead are

$$
\begin{aligned}
& \mathcal{J}_{R}^{+}=\left(\begin{array}{ccc}
0 & \Gamma_{R}^{f} & \Gamma_{R}^{s} f_{R}^{-} \\
0 & 0 & 0 \\
0 & 0 & 0
\end{array}\right), \\
& \mathcal{J}_{R}^{-}=\left(\begin{array}{ccc}
0 & 0 & 0 \\
0 & 0 & 0 \\
\Gamma_{R}^{s} f_{R}^{+} & 0 & 0
\end{array}\right) .
\end{aligned}
$$

In our considerations, $\Gamma_{\alpha}^{s} f_{\alpha}^{-}$gives the bottleneck for transport; therefore we use $f_{\alpha}^{+}=1-f_{\alpha}^{-}$and expand to lowest order in $\Gamma_{\alpha}^{s} f_{\alpha}^{-}$to obtain the current in the right lead,

$$
I_{R}=-e \frac{\left(\Gamma_{L}^{f}+\Gamma_{L}^{s}\right) \Gamma_{R}^{s} f_{R}^{-}+\left(\Gamma_{L}^{f}-\Gamma_{R}^{s}\right) \Gamma_{L}^{s} f_{L}^{-}}{\Gamma_{L}^{s}+\Gamma_{R}^{s}}
$$

In a CB situation with $f_{R}^{-} \gg f_{L}^{-}$and for large asymmetry between the couplings to the leads, $\Gamma_{L}^{s} \gg \Gamma_{R}^{s}$, Eq. (B3) simplifies to $I_{R}=-e \Gamma_{R}^{s} f_{R}^{-}\left(1+\Gamma_{L}^{f} / \Gamma_{L}^{s}\right)$, in agreement with the findings by Belzig [10]. In the case of IB where $\Gamma_{R}^{s}=0$, the current becomes $I_{R}=-e \Gamma_{L}^{f} f_{L}^{-}$. Calculations similar to those for the current yield the Fano factor

$$
F_{n v}=1+\frac{2 \Gamma_{L}^{f}}{\Gamma_{L}^{s}+\Gamma_{R}^{s}} .
$$

Notice that $F_{n v} \geqslant 1$ always holds. The expression simplifies to $F_{n v}=1+2 \Gamma_{L}^{f} / \Gamma_{L}^{s}$ for CB with large asymmetry [10] and IB. One can show that in a situation where the state $|p\rangle$ has one electron less than $|f\rangle$ and $|s\rangle$ the Liouvillian and the current operators for the left lead are given again by Eqs. (B1) and (B2), respectively, upon exchanging $L \leftrightarrow R$ and $f_{\alpha}^{+} \leftrightarrow f_{\alpha}^{-}$. This corresponds to hole transport. With the same exchanges the current and Fano factor are obtained from Eqs. (B3) and (B4), respectively. Notice that this result includes also external asymmetries $\Gamma_{L} \neq \Gamma_{R}$.

\section{APPENDIX C: LIOUVILLIAN, CURRENT, AND FANO FACTOR AT THE $5_{0} \leftrightarrow 6$ RESONANCE}

The far left part of the stability diagram shown in Fig. 2 is dominated by $5 \leftrightarrow 6$ particle transitions. As seen in Table II, there exists only one configuration with six electrons given by the state $|6\rangle:=\left|6, E_{6} ; 0,0,0\right\rangle$. On the other hand, when five electrons populate the TQD a total of six configurations is possible. In particular, for $b<0$ the ground state is the quadruplet $\left\{\left|5, E_{5_{0}} ; 1 / 2, \pm 1 / 2, \pm 1\right\rangle\right\}$ due to both orbital and spin degeneracies. The first excited state is the doublet $\left\{\left|5, E_{5_{1}} ; 1 / 2, \pm 1 / 2,0\right\rangle\right\}$ and is only spin degenerate. In this appendix we provide the explicit form of the tunneling Liouvillian $\mathcal{L}_{t}$ when the Fock space is restricted to the subspaces associated with the five- and six-particle ground states. Such a Liouvillian determines the stationary reduced density matrices $\rho^{5}\left(E_{5_{0}}\right)$ and $\rho^{6}$ when Lamb shifts are neglected. Since the six-particle ground state is a singlet, $\rho^{6}$ is just a $1 \times 1$ matrix. On the other hand, the calculation of $\rho^{5}\left(E_{5_{0}}\right)$ involves first the evaluation of the matrix elements $\rho_{L_{z} L_{z}^{\prime}}^{5 \frac{1}{2} S_{z}}=\left\langle 5, E_{5_{0}} ; 1 / 2, S_{z}, L_{z}\left|\rho^{\infty}\right| 5, E_{5_{0}} ; 1 / 2, S_{z}, L_{z}^{\prime}\right\rangle$ and then a summation over $S_{z}: \rho_{L_{z} L_{z}^{\prime}}^{5}:=\sum_{S_{z}} \rho_{L_{z} L_{z}}^{5 \frac{1}{2} S_{z}}$. Hence, $\rho^{5}\left(E_{5_{0}}\right)$ is a $2 \times 2$ matrix in a basis spanned by the vectors $\left|5_{0}^{+}\right\rangle$and $\left|5_{0}^{-}\right\rangle$, where plus and minus symbols refer to the associated values of the angular momentum. Notice that coherences between states with the same particle number but different angular momenta have to be considered. We choose the basis $\left\{\left|5_{0}^{+}\right\rangle\left\langle 5_{0}^{+}|,| 5_{0}^{-}\right\rangle\left\langle 5_{0}^{-}|| 6,\right\rangle\left\langle 6|,| 5_{0}^{+}\right\rangle\left\langle 5_{0}^{-}|,| 5_{0}^{-}\right\rangle\left\langle 5_{0}^{+}\right|\right\}$. Then the tunneling Liouvillian can be written as

$$
\mathcal{L}_{t}=\frac{\Gamma_{0}}{3}\left(\begin{array}{ccccc}
-f_{R}^{+}-f_{L}^{+} & 0 & 2\left(f_{R}^{-}+f_{L}^{-}\right) & \frac{1}{2} X & \frac{1}{2} X^{*} \\
0 & -f_{R}^{+}-f_{L}^{+} & 2\left(f_{R}^{-}+f_{L}^{-}\right) & \frac{1}{2} X & \frac{1}{2} X^{*} \\
f_{R}^{+}+f_{L}^{+} & f_{R}^{+}+f_{L}^{+} & -4\left(f_{R}^{-}+f_{L}^{-}\right) & -X & -X^{*} \\
\frac{1}{2} X^{*} & \frac{1}{2} X^{*} & 2\left(1+X^{*}\right) & -f_{R}^{+}-f_{L}^{+} & 0 \\
\frac{1}{2} X & \frac{1}{2} X & 2(1+X) & 0 & -f_{R}^{+}-f_{L}^{+}
\end{array}\right),
$$

with the short notation $X=e^{-i \frac{2 \pi}{3}} f_{R}^{+}+e^{i \frac{2 \pi}{3}} f_{L}^{+}$. Note that the phases $e^{ \pm i \frac{2 \pi}{3}}$ arise from changing from the position to angular momentum basis. The solution of the equation $\mathcal{L}_{t} \rho^{\infty}=0$, together with the constraint $\operatorname{Tr}_{\mathrm{TQD}}\left\{\rho^{\infty}\right\}=1[$ with 
$\left.\operatorname{Tr}_{\mathrm{TQD}}=(1,1,1,0,0)\right]$, yields the stationary density matrix vector

$$
\rho^{\infty}=\frac{1}{D}\left(\begin{array}{c}
f_{R}^{+} f_{L}^{-}+f_{L}^{+} f_{R}^{-} \\
f_{R}^{+} f_{L}^{-}+f_{L}^{+} f_{R}^{-} \\
f_{R}^{+} f_{L}^{+} \\
\frac{1}{2}\left(f_{R}^{+}-f_{L}^{+}\right)\left[\frac{f_{R}^{+}-f_{L}^{+}}{f_{R}^{+}+f_{L}^{+}}-i \sqrt{3}\right] \\
-\frac{1}{2}\left(f_{R}^{+}-f_{L}^{+}\right)\left[\frac{f_{R}^{+}-f_{L}^{+}}{f_{R}^{+}+f_{L}^{+}}+i \sqrt{3}\right]
\end{array}\right)
$$

with $D=2\left(f_{R}^{+} f_{L}^{-}+f_{L}^{+} f_{R}^{-}\right)+f_{R}^{+} f_{L}^{+}$.

The current operators for the right lead can be calculated along the same lines and read in the same basis

$$
\mathcal{J}^{+}=\frac{\Gamma_{0}}{3} f_{R}^{-}\left(\begin{array}{ccccc}
0 & 0 & 2 & 0 & 0 \\
0 & 0 & 2 & 0 & 0 \\
0 & 0 & 0 & 0 & 0 \\
0 & 0 & -2 e^{i \frac{2 \pi}{3}} & 0 & 0 \\
0 & 0 & -2 e^{-i \frac{2 \pi}{3}} & 0 & 0
\end{array}\right), \quad \mathcal{J}^{-}=\frac{\Gamma_{0}}{3} f_{R}^{+}\left(\begin{array}{ccccc}
0 & 0 & 0 & 0 & 0 \\
0 & 0 & 0 & 0 & 0 \\
1 & 1 & 0 & -e^{-i \frac{2 \pi}{3}} & -e^{i \frac{2 \pi}{3}} \\
0 & 0 & 0 & 0 & 0 \\
0 & 0 & 0 & 0 & 0
\end{array}\right),
$$

which results in the current through the right lead

$$
I=-e \frac{\Gamma_{0}}{3} \frac{\left(f_{L}^{+}-f_{R}^{+}\right) f_{R}^{+} f_{L}^{+}}{\left(f_{R}^{+}+f_{L}^{+}\right)\left[2\left(f_{R}^{+} f_{L}^{-}+f_{L}^{+} f_{R}^{-}\right)+f_{R}^{+} f_{L}^{+}\right]}
$$

and the Fano factor

$$
\begin{aligned}
F_{n v}= & \frac{2\left(3 f_{L}^{-}+1\right)\left(f_{R}^{+}\right)^{2}\left[\left(f_{L}^{+}\right)^{2}+2 f_{L}^{+} f_{L}^{-}-2 f_{L}^{-} f_{R}^{+}\right]}{\left(f_{L}^{+}+f_{R}^{+}\right)\left(f_{L}^{+} f_{R}^{-}+f_{L}^{+}+2 f_{L}^{-} f_{R}^{+}\right)^{2}}+\frac{5\left(f_{L}^{+}\right)^{2}+5 f_{L}^{+} f_{R}^{+}+3\left(f_{R}^{+}\right)^{2}}{\left(f_{L}^{+}+f_{R}^{+}\right)^{2}}+\frac{6 f_{L}^{-} f_{R}^{+}}{f_{R}^{+}-f_{L}^{+}} \\
& -\frac{f_{R}^{+}\left[\left(f_{L}^{+}\right)^{2}\left(8 f_{L}^{-}+f_{R}^{+}+4\right)\right]}{\left(f_{L}^{+}+f_{R}^{+}\right)^{2}\left(f_{L}^{+} f_{R}^{-}+f_{L}^{+}+2 f_{L}^{-} f_{R}^{+}\right)}+\frac{f_{R}^{+}\left[4 f_{L}^{+} f_{L}^{-} f_{R}^{+}+4 f_{L}^{-}\left(f_{R}^{+}\right)^{2}\right]}{\left(f_{L}^{+}+f_{R}^{+}\right)^{2}\left(f_{L}^{+} f_{R}^{-}+f_{L}^{+}+2 f_{L}^{-} f_{R}^{+}\right)} .
\end{aligned}
$$

Away from resonance lines, the Fermi functions can be approximated by step functions. Setting $f_{L}^{+}=f_{R}^{-}=1, f_{L}^{-}=f_{R}^{+}=0$ (valid at positive electrochemical potential $e V_{\mathrm{b}}$ ), the Liouvillian simplifies to

$$
\mathcal{L}_{t}=\frac{\Gamma_{0}}{3}\left(\begin{array}{ccccc}
-1 & 0 & 2 & \frac{Y}{2} & \frac{Y^{*}}{2} \\
0 & -1 & 2 & \frac{Y}{2} & \frac{Y^{*}}{2} \\
1 & 1 & -4 & -Y & -Y^{*} \\
\frac{Y^{*}}{2} & \frac{Y^{*}}{2} & 2\left(1+Y^{*}\right) & -1 & 0 \\
\frac{Y}{2} & \frac{Y}{2} & 2(1+Y) & 0 & -1
\end{array}\right),
$$

where $Y=e^{i 2 \pi / 3}$. Similarly, the density matrix becomes

$$
\rho^{\infty}=\frac{1}{2}\left(1,1,0, e^{-i 2 \pi / 3}, e^{i 2 \pi / 3}\right)^{\top},
$$

which shows full occupation of the five-particle ground states and an empty six-particle state. Therefore the current through the system is blocked. Since the $5_{0}$ ground states block the current, one speaks of a ground-state blockade [18]. The stability diagram for the current [Fig. 2(a)] shows how the current gets strongly suppressed at the ground-state blockade and features only a small line of finite current at the ground-state resonance, $E_{6}\left(V_{\mathrm{g}}, V_{\mathrm{b}}\right)=E_{5_{0}}\left(V_{\mathrm{g}}, V_{\mathrm{b}}\right)$.

As expected, the Fano factor is Poissonian, $F=1$, in the region of the six-particle Coulomb diamond and diverges for $V_{\mathrm{b}} \rightarrow 0$. In the region of the ground-state blockade it has the super-Poissonian value of $F_{n v}=5 / 3$, in agreement with the full numerical results shown in Fig. 2(c).

\section{APPENDIX D: CURRENT AND FANO FACTOR FOR A MINIMAL MODEL AT $\xi=\mathbf{- 7 . 5 | b |}$}

A striking feature on the left side of the stability diagram, a Fano factor of $F=4 / 3$, cannot be obtained considering a minimal model using only the five- and six-particle states. It appears at bias and gate voltages where $f_{R}^{-}\left(E_{5_{0}}-E_{4_{0}}\right)$ overcomes $f_{R}^{+}\left(E_{6}-E_{5_{0}}\right)$ and the transition to the triplet of ground states with four particles becomes the new bottleneck of transport. At $\xi=$ $-7.5|b|$, this happens at $e V_{\mathrm{b}}=\left(E_{6}-E_{4_{0}}\right)=V=2|b|$. A minimal model can be written in the basis $\{|6\rangle\langle 6|| d\rangle,\langle d|| c\rangle,\langle c|| 4\rangle,\langle 4|\}$, with the coupled state $|c\rangle$, decoupled state $|d\rangle$, and channel $|4\rangle$ associated with the triplet $\left.\left\{4, E_{4_{0}} ; 1, S_{z}, 0\right\rangle\right\}$. The Liouvillian and 
current superoperators are

$$
\begin{gathered}
\mathcal{L}=\left(\begin{array}{cccc}
-\Gamma_{R, 65}^{c}-\Gamma_{R, 65}^{d} & 0 & \Gamma_{L, 65}^{c} & 0 \\
\Gamma_{R, 65}^{d} & -\Gamma_{R, 54}^{d} f_{R}^{-} & 0 & \Gamma_{L, 54}^{d}+\Gamma_{R, 54}^{d} \\
\Gamma_{R, 65}^{c} & 0 & -\Gamma_{L, 65}^{c} & \Gamma_{L, 54}^{c}+\Gamma_{R, 54}^{c} \\
0 & \Gamma_{R, 54}^{d} f_{R}^{-} & 0 & -\Gamma_{L, 54}^{d}-\Gamma_{R, 54}^{d}-\Gamma_{L, 54}^{c}-\Gamma_{R, 54}^{c}
\end{array}\right), \\
\mathcal{J}^{+}=\left(\begin{array}{ccccc}
0 & 0 & 0 & 0 \\
\Gamma_{R, 65}^{d} & 0 & 0 & 0 \\
\Gamma_{R, 65}^{c} & 0 & 0 & 0 \\
0 & \Gamma_{R, 54}^{d} f_{R}^{-} & 0 & 0
\end{array}\right), \mathcal{J}^{-}=\left(\begin{array}{cccc}
0 & 0 & 0 & 0 \\
0 & 0 & 0 & \Gamma_{R, 54}^{d} \\
0 & 0 & 0 & \Gamma_{R, 54}^{c} \\
0 & 0 & 0 & 0
\end{array}\right)
\end{gathered}
$$

where $f_{R}^{-}$is the Fermi function between the five- and four-particle ground states at the right lead and is responsible for the bottleneck process. To lowest order in this Fermi function, the current and Fano factor read

$$
\begin{aligned}
I & =-e \Gamma_{R, 54}^{d} f_{R}^{-} \frac{\Gamma_{L, 54}^{c}\left(\Gamma_{R, 65}^{c}+2 \Gamma_{R, 65}^{d}\right)+\Gamma_{R, 54}^{c}\left(\Gamma_{R, 65}^{c}+\Gamma_{R, 65}^{d}\right)+\Gamma_{L, 54}^{d} \Gamma_{R, 65}^{d}}{\Gamma_{R, 65}^{d}\left(\Gamma_{L, 54}^{c}+\Gamma_{R, 54}^{c}+\Gamma_{L, 54}^{d}+\Gamma_{R, 54}^{d}\right)}, \\
F_{n v} & =\frac{\Gamma_{L, 54}^{c}\left[2\left(\Gamma_{R, 65}^{c}\right)^{2}+5 \Gamma_{R, 65}^{c} \Gamma_{R, 65}^{d}+4\left(\Gamma_{R, 65}^{d}\right)^{2}\right]+\Gamma_{R, 54}^{c}\left[2\left(\Gamma_{R, 65}^{c}\right)^{2}+3 \Gamma_{R, 65}^{c} \Gamma_{R, 65}^{d}+\left(\Gamma_{R, 65}^{d}\right)^{2}\right]+\Gamma_{L, 54}^{d}\left(\Gamma_{R, 65}^{d}\right)^{2}}{\Gamma_{R, 65}^{d}\left[\Gamma_{L, 54}^{c}\left(\Gamma_{R, 65}^{c}+2 \Gamma_{R, 65}^{d}\right)+\Gamma_{R, 54}^{c}\left(\Gamma_{R, 65}^{c}+\Gamma_{R, 65}^{d}\right)+\Gamma_{L, 54}^{d} \Gamma_{R, 65}^{d}\right]} .
\end{aligned}
$$

The rate matrix for the $5_{0} \leftrightarrow 4_{0}$ transitions in the angular momentum basis reads $\left(\mathcal{R}_{\alpha}\right)_{\ell \ell^{\prime}}^{5_{0} \leftrightarrow 4_{0}}=\frac{1}{2} \sum_{\sigma, \tau}\left\langle 5, E_{5_{0}}\right.$; $\left.\frac{1}{2}, \sigma, \ell\left|d_{\alpha \tau}^{\dagger} \mathcal{P}_{4, E_{4}} d_{\alpha \tau}\right| 5, E_{5_{0}} ; \frac{1}{2}, \sigma, \ell^{\prime}\right\rangle$, where $\mathcal{P}_{N E}=\sum_{S_{z}, L_{z}}\left|N, E ; S, S_{z}, L_{z}\right\rangle\left\langle N, E ; S, S_{z}, L_{z}\right|$ is the projector on the $N$-particle level with energy $E$ and spin $S$ and $\ell, \ell^{\prime}= \pm 1$. Under the bias and gate voltage conditions considered here, the system still remains in the interference ground-state blocking associated with the $5_{0} \leftrightarrow 6$ transition. The corresponding coupled-decoupled basis introduced shortly above Eq. (13) is thus the most convenient representation. With the help of the eigenstates listed in Table II and the Wigner-Eckart theorem one calculates $\Gamma_{\alpha, 54}^{d}=\frac{3}{2} \Gamma R_{\alpha}^{f}$ and $\Gamma_{\alpha, 54}^{c}=\frac{3}{2} \Gamma R_{\alpha}^{s}$, with $R_{\alpha}^{f / s}$ as given below Eq. (13). Finally, by substitution into Eq. (D3), one obtains $I=-e \Gamma f_{R}^{-} / 4$ and $F=4 / 3$.

[1] R. Landauer, Nature (London) 392, 658 (1998).

[2] S. Hershfield, J. H. Davies, P. Hyldgaard, C. J. Stanton, and J. W. Wilkins, Phys. Rev. B 47, 1967 (1993).

[3] U. Hanke, Y. M. Galperin, K. A. Chao, and N. Zou, Phys. Rev. B 48, 17209 (1993).

[4] A. N. Korotkov, Phys. Rev. B 49, 10381 (1994).

[5] A. Nauen, I. Hapke-Wurst, F. Hohls, U. Zeitler, R. J. Haug, and K. Pierz, Phys. Rev. B 66, 161303 (2002).

[6] D. A. Bagrets and Y. V. Nazarov, Phys. Rev. B 67, 085316 (2003).

[7] A. Thielmann, M. H. Hettler, J. König, and G. Schön, Phys. Rev. B 68, 115105 (2003).

[8] E. V. Sukhorukov, G. Burkard, and D. Loss, Phys. Rev. B 63, 125315 (2001).

[9] A. Cottet and W. Belzig, Europhys. Lett. 66, 405 (2004).

[10] W. Belzig, Phys. Rev. B 71, 161301 (2005).

[11] G. Kießlich, A. Wacker, and E. Schöll, Phys. Rev. B 68, 125320 (2003).

[12] C. W. Groth, B. Michaelis, and C. W. J. Beenakker, Phys. Rev. B 74, 125315 (2006).

[13] C. Pöltl, C. Emary, and T. Brandes, Phys. Rev. B 80, 115313 (2009).

[14] G. Schaller, G. Kießlich, and T. Brandes, Phys. Rev. B 80, 245107 (2009).

[15] M. Benito, M. Niklas, G. Platero, and S. Kohler, Phys. Rev. B 93, 115432 (2016).
[16] M. Korkusinski, I. P. Gimenez, P. Hawrylak, L. Gaudreau, S. A. Studenikin, and A. S. Sachrajda, Phys. Rev. B 75, 115301 (2007).

[17] T. Kostyrko and B. R. Bułka, Phys. Rev. B 79, 075310 (2009).

[18] A. Donarini, G. Begemann, and M. Grifoni, Nano Lett. 9, 2897 (2009).

[19] H. Pan, M. G. House, X. Hao, and H. W. Jiang, Appl. Phys. Lett. 100, 263109 (2012).

[20] M. Seo, H. K. Choi, S.-Y. Lee, N. Kim, Y. Chung, H.-S. Sim, V. Umansky, and D. Mahalu, Phys. Rev. Lett. 110, 046803 (2013).

[21] S. Andergassen, Nature (London) 495, 321 (2013).

[22] S.-Y. Lee and Y. Chung, Phys. Rev. B 87, 045302 (2013).

[23] M. Kotzian, F. Gallego-Marcos, G. Platero, and R. J. Haug, Phys. Rev. B 94, 035442 (2016).

[24] L. Gaudreau, S. A. Studenikin, A. S. Sachrajda, P. Zawadzki, A. Kam, J. Lapointe, M. Korkusinski, and P. Hawrylak, Phys. Rev. Lett. 97, 036807 (2006).

[25] H. H. Weitering, Nat. Nanotechnol. 9, 499 (2014).

[26] F. Domínguez, G. Platero, and S. Kohler, Chem. Phys. 375, 284 (2010).

[27] I. Weymann, B. R. Bułka, and J. Barnaś, Phys. Rev. B 83, 195302 (2011).

[28] K. Wrześniewski and I. Weymann, Phys. Rev. B 92, 045407 (2015).

[29] A. Donarini, G. Begemann, and M. Grifoni, Phys. Rev. B 82, 125451 (2010). 
[30] C.-Y. Hsieh, Y.-P. Shim, M. Korkusinski, and P. Hawrylak, Rep. Prog. Phys. 75, 114501 (2012).

[31] C. Flindt, T. Novotný, A. Braggio, and A.-P. Jauho, Phys. Rev. B 82, 155407 (2010).

[32] F. Kaiser and S. Kohler, Ann. Phys. (Berlin, Ger.) 16, 702 (2007).

[33] M. G. Schultz and F. von Oppen, Phys. Rev. B 80, 033302 (2009).
[34] F. Bodoky, W. Belzig, and C. Bruder, Phys. Rev. B 77, 035302 (2008).

[35] W. Belzig and A. Bednorz, Phys. Status Solidi B 251, 1945 (2014).

[36] J. König, J. Schmid, H. Schoeller, and G. Schön, Phys. Rev. B 54, 16820 (1996).

[37] A. Messiah, Quantum Mechanics (North-Holland, Amsterdam, 1961), Vol. 2. 